\title{
Japanese Society of Gastroenterological Surgery Article Series Disclosure Index
}

The Annals of Surgical Oncology ensures balance, independence, objectivity, and scientific rigor in all its published articles. Authors are expected to disclose any significant financial interest or other relationship(s) involving themselves with any proprietary entity producing health care goods or services related to the content of the article (Table 1).

TABLE 1 Disclosure index for Japanese Society of Gastroenterological Surgery article series

\begin{tabular}{lll}
\hline Article title & $\begin{array}{l}\text { Lead } \\
\text { author }\end{array}$ & Disclosure \\
\hline
\end{tabular}

8q24 Polymorphisms and Diabetes Mellitus Regulate Apolipoprotein A-IV in Colorectal Carcinogenesis

Neurotrophin Receptor-Interacting Melanoma AntigenEncoding Gene Homolog is Associated with Malignant Phenotype of Gastric Cancer

Expression of PD-L1 and HLA Class I in Esophageal Squamous Ito, Shuhei Cell Carcinoma: Prognostic Factors for Patient Outcome

FIB-4 Index is a Predictor of Background Liver Fibrosis and Long-Term Outcomes After Curative Resection of Hepatocellular Carcinoma

Parenchyma-Sparing Hepatectomy with Vascular Reconstruction Techniques for Resection of Colorectal Liver Metastases with Major Vascular Invasion

Clinical Significance of Extramural Tumor Deposits in the Lateral Pelvic Lymph Node Area in Low Rectal Cancer: A Retrospective Study at Two Institutions

Risk Factors for the Development of Desmoid Tumor After Colectomy in Patients with Familial Adenomatous Polyposis: Multicenter Retrospective Cohort Study in Japan

Significance of Vascular Resection and Reconstruction in Surgery for Hilar Cholangiocarcinoma: With Special Reference to Hepatic Arterial Resection and Reconstruction

Vascularity of Intrahepatic Cholangiocarcinoma on Computed Tomography is Predictive of Lymph Node Metastasis

Feasibility of Laparoscopic Radical Gastrectomy for Gastric Cancer of Clinical Stage II or Higher: Early Outcomes in a Phase II Study (KUGC04)

Aggressive Hemihepatectomy Combined with Resection and Reconstruction of Middle Hepatic Vein for Intrahepatic Cholangiocarcinoma

Impact of Sarcopenic Obesity on Surgical Site Infection after Laparoscopic Total Gastrectomy

Perioperative Adiponectin Measurement is Useful for Prediction of Postoperative Infection in Patients with Colorectal Cancer

Mimori, Koshi

Kanda, Mitsuro

Okamura, Yukiyasu

Ko, Saiho

Shimada,

Hinoi,

Takao

Yamamoto, Yusuke

Okabe, Hiroshi

Kokudo, Norihiro

Tsunoda, Shigeru

Yamamoto, Hiroshi
Disclosure. The author has no commercial interests related to the subject of the study, and the study did not receive any external financial or material support.

Disclosure. The authors have no commercial interests related to the subject of the study, and the study did not receive any external financial or material support.

Disclosure. The authors have no commercial interests related to the subject of the study, and the study did not receive any external financial or material support.

Disclosure. The authors have no commercial interests related to the subject of the study, and the study did not receive any external financial or material support.

Disclosure. The authors have no commercial interests related to the subject of the study, and the study did not receive any external financial or material support.

Yoshifumi

Disclosure. The authors have no commercial interests related to the subject of the study, and the study did not receive any external financial or material support.

Disclosure. The authors have no commercial interests related to the subject of the study, and the study did not receive any external financial or material support.

Matsuyama, Disclosure. The authors have no commercial interests related to Ryusei the subject of the study, and the study did not receive any external financial or material support.
Disclosure. The authors have no commercial interests related to the subject of the study, and the study did not receive any external financial or material support.

Disclosure. The authors have no commercial interests related to the subject of the study, and the study did not receive any external financial or material support.

Disclosure. The authors have no commercial interests related to the subject of the study, and the study did not receive any external financial or material support.

Disclosure. The authors have no commercial interests related to the subject of the study, and the study did not receive any external financial or material support.

Disclosure. The authors have no commercial interests related to the subject of the study, and the study did not receive any external financial or material support. 